\title{
Orientaciones de meta en Educación Física y nivel de actividad físico- deportiva en estudiantes mexicanos
}

\section{Goal Orientation in Physical Education and Physical Activity Level on Mexican Students}

Recibido: febrero 8 de 2012 | Revisado: febrero 26 de 2012 | Aceptado: mayo 1 de 2012

\author{
FrANCISCO RUIZ-JUAN* \\ Universidad de Murcia, España \\ Maurice PiÉron ** \\ Universidad de Liéja, Bélgica
}

SICI: 1657-9267(201303)12:1<235:OMEFNA >2.0.TX;2-W

Para citar este artículo: Ruiz-Juan, F. \& Piéron, M. (2013). Orientaciones de meta en Educación Física y nivel de actividad físico-deportiva en estudiantes mexicanos. Universitas Psychologica, 12(1), 235-247.

* fruizj@um.es

***mpieron@ulg.ac.be
RESUMEN

Se evalúan las orientaciones de meta en Educación Física de estudiantes mexicanos, así como las diferencias según sexo, edad, implicación en actividad físico-deportiva y nivel de práctica habitual. Se administró un cuestionario por autorreporte a 1.083 estudiantes mexicanos regiomontanos de secundaria (12-16 años; error $\pm 3 \%, \mathrm{IC}=95.5 \%)$, por muestreo polietápico estratificado por afijación proporcional y conglomerados. El análisis factorial confirmatorio apoyó el modelo teórico hipotetizado de dos factores para TEOSQ-12EF, mostrando excelente bondad de ajuste del modelo original. Tanto chicos como chicas tienen, principalmente, una orientación a la tarea, estando los chicos más orientados al ego que las chicas. Según aumenta el nivel de actividad física, incrementa la orientación al ego y a la tarea. Palabras clave autores

Orientación de meta, Educación Física, características psicológicas.

Palabras clave descriptores

Motivación del estudiante, compromiso de aprendizaje, análisis de la tarea.

\section{A B S T R AC T}

Are assessed goal orientations in physical education of Mexican students and differences by sex, age, involvement in regular exercise and practice level? Self-administered questionnaire about habitual physical activity was administered in 1083 high school Mexican students (12/16 years; error $\pm 3 \%, \mathrm{CI}=95.5 \%$ ) using a multistage sampling stratification by proportional affix and by conglomerates. Factor analysis confirmatory supported the hypothesized theoretical model of two factors for TEOSQ-12EF showing excellent goodness of fit of the original model. Both boys and girls have primarily a task orientation, with boys more ego-oriented than girls. With increasing levels of physical activity, increases task and ego orientations. Key words authors

Goal orientation, Physical Education, psychological characteristics.

Key words plus

Student Motivation, Learner Engagement, Task Analysis. 


\section{Introducción}

La orientación de meta tiene su origen en la investigación pedagógica. Nicholls, Patashnick y Nolen (1985) analizaron los niveles de orientación al ego o la tarea en alumnos de la enseñanza secundaria, intentando determinar lo que estos consideran como muy importante en la escuela. Desarrollaron un cuestionario para evaluar la opinión de los estudiantes sobre los motivos de sus estudios, las metas personales dentro de la escuela y la satisfacción con el aprendizaje escolar, además, examinaron las causas percibidas de éxito y la percepción de competencia (o habilidad). Los datos pusieron de relieve cuatro factores, que corresponden a dos maneras muy diferentes de considerar valores y beneficios que deberían derivar de la educación: (1) la educación representa un medio para alcanzar un objetivo: riqueza y estatus social; (2) la educación es un objetivo por sí mismo: permitir al individuo controlar, reforzar el deseo de educarse, perfeccionarse, convertirse en un ciudadano responsable y capaz de ponerse al servicio de la comunidad. Mientras que la primera concepción es característica de orientación al ego, la segunda perspectiva de la educación se relacionada estrechamente con la tarea.

En el ámbito deportivo, autores como Duda (1989a) y Nicholls (1992) trazaron un paralelo de esta teoría. Los beneficios materiales de participación deportiva (gloria, dinero) se asocian al ego, mientras que la orientación a la tarea corresponde a una búsqueda de desarrollo personal y control.

Un elemento clave en la teoría de Nicholls (1992) está en que los objetivos de logro están relacionados con estas diferentes concepciones de los objetivos que se persiguen al adquirir una habilidad. Existen dos principales objetivos de logro que implican distintas concepciones de competencia: objetivos orientados hacia la tarea y objetivos centrados en el ego. En el primer caso, una mejora subjetiva propia del resultado o una mejor prestación de lo esperado produce sentimientos de competencia y éxito. La percepción del éxito, en alguien orientado a la tarea, se basa en características propias de la habilidad en sí. Implícitamente, existe preocupación por el desarrollo personal, entendiendo el éxito como mejora de la competencia personal, generando una importante motivación intrínseca, siendo la mejor recompensa el logro de sí mismo y, por lo tanto, tienen escasa preocupación por el fracaso al percibir sus propios errores como parte del aprendizaje (Duda, 1993; Duda \& Balaguer, 2007).

En los objetivos centrados en el ego, la percepción del éxito procede de una comparación favorable de sus capacidades con las de otros. En definitiva, su motivación es extrínseca al concebir el aprendizaje basado en el logro de reconocimiento público de su superioridad. Esto puede provocar conductas de inadaptación, resistencia al esfuerzo y evitación de actividades que supongan un reto, por tanto, conductas de abandono de la práctica y deterioro de la ejecución son algunas de sus consecuencias al no confiar en la propia capacidad o competencia (Duda \& Balaguer, 2007; Nicholls, 1992).

El tipo de orientación (tarea-ego) a la que cada persona tiende varía según diversos determinantes. Nicholls (1992) destaca la influencia de madurez cognoscitiva, disposiciones personales y determinantes específicos a la situación. Otras variables influyentes reveladas por la investigación son sexo, cultura y edad de los participantes.

Investigaciones realizadas desde la perspectiva de meta ponen de manifiesto que en el proceso de socialización chicos y chicas, tanto en contextos de logro en deporte como en Educación Física, sufren influencias sobre el desarrollo de sus orientaciones de meta. Es decir, muestran diferencias en su disposición a estar orientados al ego y a la tarea, relevando que los chicos de estas edades presentan una mayor orientación al ego que las chicas (Van Der Horst, Paw, Twisk \& Van Mechelen, 2007). Según la edad, los niños de 9-11 años tienden a favorecer objetivos centrados en la tarea, mientras que los jóvenes adolescentes (12-14 años) se orientaban más hacia objetivos centrados en el ego (Piéron \& RuizJuan, 2010; Ruiz-Juan, Piéron \& Zamarripa, 2011).

En este alumnado una orientación hacia el ego guarda relación con el deseo de demostrar superioridad sobre los iguales, asociado a un conjunto de creencias y valores que corresponden a una necesidad del niño adolescente de causar impresión sobre los iguales y el profesor (Baena, Flores \& Barbero, 
2007). En alumnado de 15 años, los chicos obtienen resultados de ego significativamente superiores a las chicas (Chinchilla, Escribano, Romero \& López, 2008) y, además, consideran que obtener ocasiones para entrar en competición constituía un objetivo que la educación física debía garantizar (Walling, Duda \& Chi, 1993).

La perspectiva de metas de logro es considerada como marco teórico apropiado para analizar la motivación deportiva, ya que contempla como determinantes de la motivación la percepción subjetiva que los sujetos tienen de la situación deportiva (Duda, 1993; Duda \& Balaguer, 2007). La actividad deportiva es una situación de exigencia de logro, en la que el deportista intenta alcanzar una meta y donde es importante demostrar la competencia. Además, el deporte suele ser una actividad libre, por lo que el inicio, mantenimiento y abandono de la práctica físico-deportiva son aspectos interesantes para ser abordados desde la perspectiva de las metas de logro (Piéron \& Ruiz-Juan, 2010).

Generalmente, los jóvenes deportistas se orientan más hacia la tarea que hacia el ego. Williams y Gill (1995) indican que la orientación a la tarea influye directamente sobre la competencia percibida, esfuerzo y motivación intrínseca. Además, esta última desempeña un papel mediador entre la percepción de competencia y la orientación a la tarea (Cervelló, Jiménez, Del Villar, Ramos \& Santa Rosa, 2004; Wang, Chatzisarantis, Spray \& Biddle, 2002; White \& Duda, 1994).

Varios autores ponen de relieve las relaciones existentes entre las orientaciones de meta, la continuidad en la actividad, intensidad o dinamismo con que se aplican comportamientos y autopercepciones, tanto en deportistas de competición como en personas que se dedican a actividades deportivas de ocio (Cervelló, Moreno, Alonso \& Iglesias, 2006; Duda, 1989b; Molero, Ortega, Valiente \& Zagalaz, 2010; Ries, 2011). El énfasis sobre la orientación a la tarea favorece sensaciones de competencia y esfuerzo, mientras que un objetivo destinado simplemente a superar a otros puede conducir a percepciones de incompetencia y menor continuación de la actividad (Duda, 1992; Nicholls, 1992; Piéron \& Ruiz-Juan, 2010).
Esta teoría se sitúa en los enfoques contemporáneos de las teorías motivacionales de metas de logro (Ames, 1984; Castillo, Balaguer \& Duda, 2000; Nicholls, 1984). Una de sus ventajas es que ha sido ampliamente aplicada al terreno deportivo, desarrollando instrumentos de medida específicos para este entorno. Se basa en la existencia de dos objetivos de logro, surgiendo metas de maestría (orientación a la tarea) y metas de competitividad (orientación al ego). Cuando la meta es de maestría (tarea), los sujetos juzgan su nivel de capacidad basándose en un proceso de comparación con ellos mismos, mientras que cuando la meta es de competitividad (ego), los individuos juzgan si son o no competentes, comparándose con los demás (Cervelló, Escartí \& Balagué, 1999; Nicholls, 1984; Roberts \& Treasure, 1992).

Una orientación hacia la tarea corresponde a elecciones de tareas más estimulantes, presentando un desafío más elevado. Esto sugiere que la orientación de los objetivos puede influir en las clases de Educación Física (Gómez, Ruiz, García, Baena \& Granero, 2007), de forma que la orientación hacia la tarea correspondería a mejores resultados en medidas que reflejan la acción y la reflexión del alumnado (Chinchilla et al., 2008). Los alumnos para quienes los criterios de éxito o competencia se erigen en referencia, tomando como base la propia habilidad, emplearían procesos cognoscitivos que favorecen el aprendizaje. En el medio escolar, una orientación hacia el ego está relacionada con la creencia de que el éxito proviene de mostrar una habilidad superior a la media y una superación de los iguales (Treasure \& Roberts, 1994).

Diferentes estudios en el contexto de la educación física han puesto de manifiesto la relación entre las orientaciones de meta y las actitudes, la intención, la participación y el nivel de actividad física dentro y fuera de la escuela. Un reciente estudio examinó los correlatos del comportamiento de las orientaciones de meta con una medida objetiva de la actividad física en las clases de educación física. Los resultados revelaron que los estudiantes con altos valores de orientación hacia la tarea tuvieron una mayor participación en las actividades físicas vigorosas independientemente 
de su orientación al ego (Tzetzis, Goudas, Kourteissis \& Zisi, 2002).

Por otro lado, después de un programa de intervención en la clase de educación física se compararon los efectos sobre el clima motivacional, las orientaciones de meta, las actitudes hacia el ejercicio y la alimentación saludable en un grupo control y un grupo experimental. La intervención fue efectiva en la creación de un fuerte clima implicado a la tarea y una débil implicación al ego. Después de finalizar la intervención, los estudiantes del grupo control mostraron puntajes más bajos en la orientación al ego y más altos en la orientación a la tarea, además percibieron que su profesor se enfatizaba más en el involucramiento a la tarea y menos en la orientación al ego, presentando una actitud más positiva al ejercicio además de permanecer más tiempo por sesión haciendo ejercicio (Christodoulidis, Papaioannou \& Digelidis, 2001; Digelidis, Papaioannou, Laparidis \& Christodoulidis, 2003).

Asimismo, otros estudios han revelado que la orientación y la percepción del clima implicado a la tarea promueven el esfuerzo y el disfrute (o motivación intrínseca) en la clase de educación física, así como una actitud e intención positiva y participación en el ejercicio (Escarti \& Gutiérrez, 2001; Papaioannou, Marsh \& Theodorakis, 2004; Wang, Biddle \& Elliot, 2007), sugiriendo que son fuertes predictores positivos del deporte y la participación futura del ejercicio fuera de la escuela (Papaioannou et al., 2004).

Varios estudios convergen en la existencia de una relación significativa entre motivación intrínseca e intención de participar en actividades físicodeportivas. Una motivación intrínseca adecuada se asocia a una mayor probabilidad de practicar ejercicio físico habitual y continuamente (Dishman \& Sallis, 1990; Trost, Owen, Bauman, Sallis $\&$ Brown, 2002).

El control de la tarea favorece las sensaciones de competencia y esfuerzo, mientras que un objetivo destinado simplemente a superar a los otros puede conducir a percepciones de incompetencia y menor continuación de la actividad (Duda, 1992; Nicholls, 1992). La orientación hacia la tarea aparece como concepto decisivo para aumentar la motivación (Standage \& Treasure, 2002).

En un estudio con alumnado de Enseñanza Secundaria, Ferrer-Caja y Weiss (2000) probaron un modelo en el cual se preveía que las orientaciones hacia el ego y hacia la tarea, en las clases de educación física, pudieran predecir la motivación intrínseca y el sentimiento de autonomía de los participantes. Existen fuertes relaciones positivas directas que asocian orientación hacia la tarea y motivación intrínseca, mientras que las relaciones entre orientación hacia el ego y motivación intrínseca son ligeramente negativas. Además, la orientación hacia la tarea ejerce un ligero efecto indirecto sobre regulaciones motivacionales autodeterminadas por medio de percepción de competencia (Amado, Leo, Sánchez, González \& López, 2012).

El Task and Ego Orientation in Sport Questionnaire (TEOSQ) ha sido utilizado en numerosas investigaciones para evaluar este constructo psicológico. Fue desarrollado para valorar la tendencia de las personas hacia la tarea y hacia el ego en el contexto deportivo (Duda \& Nicholls, 1992), si bien es una versión modificada y adaptada al deporte del cuestionario desarrollado por Nicholls (1989) utilizado en el contexto académico.

Desde la década de los 80 del siglo XX, son numerosos los investigadores y estudios internacionales que han tratado la orientación de meta, tanto en deportistas adolescentes en muestras de habla inglesa (Duda \& Nicholls, 1992; Duda, Olson \& Templin, 1991; Lochbaum \& Roberts, 1993), como española (Balaguer, Castillo \& Tomás, 1996; Isorna, Rial, Vaquero \& Sanmartín, 2012; Sánchez et al., 2009).

En el ámbito de las clases de Educación Físi$\mathrm{ca}$, recientemente se ha validado en español el TEOSQ-12EF (Ruiz-Juan et al., 2011) para medir la orientación de meta del alumnado de Enseñanza Secundaria, siendo un instrumento fiable que reproduce los factores que se hipotetizan desde el modelo teórico de partida. Consta de 12 ítems y evalúa la tendencia del alumnado hacia una Orientación al Ego (6 ítems) y Orientación a la Tarea (6 ítems) en Educación Física. En las 
instrucciones se pide a los sujetos: "Manifiesta tu grado de acuerdo o desacuerdo, con las siguientes afirmaciones, sobre lo que sientes cuando haces Educación Física". Las respuestas se recogen en escala tipo Likert oscilando desde muy en desacuerdo (1) a muy de acuerdo (4). La consistencia interna fue de $\alpha_{1}=0.81$ para el Ego y de $\alpha_{2}=0.82$ para la Tarea. Los análisis de ítems, homogeneidad, estructura factorial, validez de constructo y de contenido y estabilidad temporal presentan aceptables valores.

La pretensión principal de este trabajo es evaluar las orientaciones de meta en Educación Física de estudiantes mexicanos y verificar si existen diferencias significativas, en las dos subescalas, según sexo, edad, implicación en actividad físicodeportiva y nivel de práctica habitual. Del mismo modo, comprobar que el TEOSQ-12EF es aplicable también en México, aplicando procedimientos confirmatorios de análisis y examinando sus propiedades psicométricas.

\section{Método}

\section{Participantes}

Se partió de la población total de estudiantes de centros de Enseñanza Secundaria del ciclo escolar agosto 2006-julio 2007 (57.069 alumnos) de Monterrey (México) (datos facilitados por Dirección de Educación Física y Deporte, Estado de Nuevo León). Para asegurar una muestra representativa (error $\pm 3 \%$, IC $=95.5 \%$ ), se empleó un muestreo polietápico estratificado por afijación proporcional y por conglomerados, considerándose: turno (matutino-vespertino), tipo de centro (público-privado), curso (primero-tercero) y sexo (chicos-chicas).

Participaron 1.083 alumnos ( 535 chicos $=49.4 \%$; 548 chicas $=50.6 \%$ ). El rango de edad estuvo comprendido entre 12-16 años $(M=13.70 ; D E=0.95)$. Se realizó entre abril-mayo de 2007. Para evaluar la estabilidad temporal del TEOSQ-12EF se recurrió a una segunda muestra de 148 alumnos (70 chicos y 78 chicas $)\left(M_{\text {edad }}=13.9\right.$ años; $\left.D E=1.88\right)$, que completó nuevamente el instrumento seis semanas después.

\section{Instrumento}

Se utilizó el TEOSQ-12EF (Ruiz-Juan et al., 2011). Se realizó un análisis de las propiedades psicométricas, con estudiantes de Monterrey, para aportar evidencias sobre su dimensionalidad y aplicando procedimientos confirmatorios de análisis. Los análisis estadísticos indican un adecuado análisis de ítems. Los valores obtenidos son $\geq 0.3$ en coeficiente de correlación corregido ítem-total, desviación típica $>1$ y todas las opciones de respuesta fueron usadas alguna vez. Los índices de asimetría y curtosis son próximos a cero y $<2$.0. El análisis de la homogeneidad no muestra solapamiento de ítems entre las diferentes dimensiones teóricas.

Se aplicaron modelos de ecuaciones estructurales para, desde una perspectiva confirmatoria, estudiar si la dimensionalización propuesta teóricamente se ajusta a los datos resultantes con la muestra empleada. La estructura factorial del TEOSQ-12EF se evaluó con AFC (AMOS 18.0), utilizando estimación de máxima verosimilitud, recurriendo a la técnica "bootstrapping" (distribución normal de datos) y a la máxima similitud (ML), procedimiento de estimación de modelos de ecuaciones estructurales que asume una distribución normal de los datos y una escala continua de ellos, ya que el coeficiente de Mardia (7.51), mayor de 2, indica falta de normalidad multivariada en los datos con lo que se violaba una de las reglas básicas del AFC. El ajuste del modelo fue evaluado con una combinación de índices de ajuste absolutos $\left(\chi^{2} / g l\right.$-ratio entre chi cuadrado y grados de libertad-, SRMR -raíz cuadrática media de residuales-, GFI -índice de ajuste comparativoy relativos (IFI -índice de ajuste incremental-, CFI -índice de ajuste comparativo-, TLI -índice Tucker Lewis-, RMSEA - error cuadrático medio de aproximación-). El modelo puesto a prueba hipotetiza la existencia de dos variables latentes independientes Orientación al Ego y Orientación a la Tarea, que subyacen a las 12 variables, y dan cuenta de las covarianzas observadas entre ellas. Los resultados muestran una excelente bondad de ajuste del modelo original (Tablas 1 y 2). 
TABLA 1

Índices de ajuste del modelo

\begin{tabular}{lccccccc}
\hline & $\chi^{2} / g l$ & GFI & CFI & IFI & TLI & RMSEA & SRMR \\
\hline Chicos & 2.89 & 0.96 & 0.97 & 0.95 & 0.96 & 0.042 & 0.038 \\
Chicas & 2.78 & 0.96 & 0.95 & 0.96 & 0.95 & 0.049 & 0.045 \\
Total & 2.82 & 0.96 & 0.96 & 0.95 & 0.96 & 0.045 & 0.041 \\
Lo deseable & $<5$ & $>0.9$ & $>0.9$ & $>0.9$ & $>0.9$ & $<0.06$ & $<0.05$ \\
\hline
\end{tabular}

$N=1.083$

Fuente: elaboración propia.

TABLA 2

Solución estandarizada (saturaciones factoriales) para el modelo de dos factores del TEOSQ-12EF

\begin{tabular}{|c|c|c|c|c|}
\hline & \multicolumn{2}{|c|}{ Orientación al Ego } & \multicolumn{2}{|c|}{ Orientación a la Tarea } \\
\hline & $\begin{array}{c}\text { Chicos } \\
(N=535)\end{array}$ & $\begin{array}{c}\text { Chicas } \\
(\mathrm{N}=548)\end{array}$ & $\begin{array}{c}\text { Chicos } \\
(N=535)\end{array}$ & $\begin{array}{c}\text { Chicas } \\
(\mathrm{N}=548)\end{array}$ \\
\hline 1.Soy el único que puede realizar una habilidad o jugar bien & 0.75 & 0.74 & & \\
\hline 3.Puedo hacerlo mejor que mis compañeros & 0.68 & 0.72 & & \\
\hline 5.Los otros no pueden hacerlo tan bien como yo & 0.78 & 0.71 & & \\
\hline 7. Otros entorpecen el juego o la actividad y yo no & 0.79 & 0.8 & & \\
\hline 9.Yo soy el que más puntúa o el que gana & 0.65 & 0.74 & & \\
\hline 11.Soy el mejor & 0.79 & 0.69 & & \\
\hline 2.Aprendo una habilidad nueva y me motiva a practicar más & & & 0.77 & 0.79 \\
\hline 4.Aprendo algo que es divertido de hacer & & & 0.7 & 0.69 \\
\hline 6.Aprendo una tarea nueva esforzándome & & & 0.68 & 0.72 \\
\hline 8.Yo trabajo y me esfuerzo mucho & & & 0.65 & 0.68 \\
\hline 10.Aprender una habilidad me hace sentir realmente bien & & & 0.71 & 0.7 \\
\hline 12.Lo hago lo mejor que puedo & & & 0.65 & 0.68 \\
\hline
\end{tabular}

Nota. $N=1.083$

$p<0.001$ en todas las saturaciones.

Fuente: elaboración propia.

La consistencia interna del TEOSQ-12EF fue evaluada con alfa de Cronbach, presentando valores aceptables (Ego $\alpha_{1}=0.76$; Tarea $\alpha_{2}=0.80$ ). La estabilidad temporal se evaluó en 148 alumnos que completaron el TEOSQ-12EF en dos ocasiones (intervalo de seis semanas). Los resultados del pretest fueron: Ego $\alpha_{1}=0.78$ y Tarea $\alpha_{2}=0.82$; los del postest fueron Ego $\alpha_{1}=0.80$ y Tarea $\alpha_{2}=0.84$. Los valores de la correlación test-retest en Ego arrojaron $r=0.77$ y en Tarea $r=0.81$.

Se analizaron las correlaciones entre las dos dimensiones del TEOSQ-12EF (coeficiente de Pearson) para evaluar la validez de constructo del cuestionario y la presencia de formas específicas de asociación. Los resultados indican que las dos di- mensiones están significativamente correlacionadas entre sí $(r=0.09, p<0.01)$.

\section{Otras variables}

Además, se utilizaron las variables sexo, edad e implicación o no en práctica habitual de actividad físico-deportiva de tiempo libre (nunca, abandono y activos). El nivel de práctica físico-deportiva habitual (índice finlandés de actividad físico-deportiva) (Raitakari et al., 1994; Telama et al., 2005), se calculó por autorreporte de cinco preguntas referentes a frecuencia, duración, intensidad, participación en deportes organizados y competiciones deportivas. Los resultados más bajos son característicos de los menos activos, mientras que las puntuaciones más 
altas son indicativas de los individuos más activos. El alfa de Cronbach demuestra alta fiabilidad del conjunto de estas variables $(\alpha=0.86)$, asemejándose a la obtenida en los trabajos citados. Acorde con los trabajos referenciados, para representar mejor los patrones de actividad, se clasifica en: vigorosa, moderada, ligera, insuficiente y sedentarios.

\section{Procedimiento}

Se pidió permiso a los centros educativos mediante carta en la que se explicaban objetivos de investigación y la forma como se realizaría, acompañando un modelo del instrumento. Este último fue autoadministrado con aplicación masiva, completado anónimamente en una jornada escolar, con consenso y adiestramiento previo de evaluadores. Los sujetos fueron informados del objetivo del estudio, voluntariedad, absoluta confidencialidad de respuestas y manejo de datos, que no había respuestas correctas o incorrectas, solicitándoles máxima sinceridad y honestidad.

\section{Análisis estadísticos}

Se realizó, con SPSS 17.0, un ANOVA para determinar las diferencias de género, edad, comportamientos de práctica e índice finlandés.

\section{Resultados}

\section{Diferencias de género y edad}

Las diferencias de género y edad, en función de las puntuaciones medias, se realizó mediante la prueba $t$, teniendo en cuenta el test de Levene para estimar la igualdad de las varianzas con un nivel de significación de $p<0.01$. En las clases de Educación Física, tanto chicos como chicas tienen, principalmente, una Orientación a la tarea, no existiendo

\section{TABLA 3}

Medias (M), desviaciones típicas (DT) y significatividad (F, p valor) del TEOSQ-12EF, diferencias por género, edad, comportamientos ante la práctica físico-deportiva e índice finlandés

\begin{tabular}{|c|c|c|c|c|c|c|c|}
\hline & & \multicolumn{3}{|c|}{ Orientación al ego } & \multicolumn{3}{|c|}{ Orientación a la tarea } \\
\hline & & $M$ & $D E$ & $F$ & $M$ & $D E$ & $F$ \\
\hline \multicolumn{8}{|l|}{ Sexo } \\
\hline & Chicos & 2.04 & 0.99 & \multirow{2}{*}{$0.30 * *$} & 3.1 & 1.01 & \multirow{2}{*}{0.03} \\
\hline & Chicas & 1.85 & 1.01 & & 3.1 & 1.02 & \\
\hline \multicolumn{8}{|l|}{ Edad } \\
\hline & 12 años & 1.94 & 1 & \multirow{4}{*}{0.32} & 3.19 & 1.07 & \multirow{4}{*}{2.56} \\
\hline & 13 años & 1.97 & 0.98 & & 3.17 & 1.03 & \\
\hline & 14 años & 1.96 & 1.03 & & 3.06 & 1.02 & \\
\hline & 15-16 años & 1.92 & 1.01 & & 3.05 & 1.01 & \\
\hline \multicolumn{8}{|c|}{ Comportamientos } \\
\hline & Nunca & 1.97 & 1.04 & \multirow{3}{*}{$6.22 *$} & 2.70 & 1.16 & \multirow{3}{*}{$22.49 * *$} \\
\hline & Abandono & 1.99 & 1 & & 3.06 & 1.02 & \\
\hline & Activos & 1.81 & 1 & & 3.18 & 0.96 & \\
\hline \multicolumn{8}{|c|}{ Índice finlandés } \\
\hline & Sedentarios & 1.83 & 1.01 & \multirow{5}{*}{$13.16^{* *}$} & 2.95 & 1.08 & \multirow{5}{*}{$8.747^{* *}$} \\
\hline & Insuficiente & 1.86 & 1.09 & & 2.99 & 0.97 & \\
\hline & Ligera & 1.9 & 0.97 & & 3.19 & 1.04 & \\
\hline & Moderada & 1.91 & 1.07 & & 3.2 & 1.01 & \\
\hline & Vigorosa & 2.28 & 1.03 & & 3.24 & 0.99 & \\
\hline
\end{tabular}

Nota. $N=1.083$

$*_{p}<0.01 ; * p<0.001$.

Fuente: elaboración propia. 
diferencias significativas $(M=3.10)$. Si que aparecieron diferencias significativas en la Orientación al Ego, resultando la media en chicos $(M=2.04)$ mayor que en chicas $(M=1.85)$, estando los chicos más orientados al ego que las chicas. No existen diferencias estadísticamente significativas en la edad (Tabla 3).

\section{Diferencias en comportamientos de práctica e índice finlandés}

Los activos muestran menor Orientación al Ego $(M=1.81)$ y mayor Orientación a la Tarea $(\mathrm{M}=3.18)$ que los inactivos (abandono y nunca). Cuando se tiene en cuenta el índice finlandés de actividad física, los sujetos con índice de práctica vigorosa tienen mayor Orientación al Ego $(M=2.28)$ y también Orientación a la Tarea $(M=3.24)$ que aquellos cuyo índice se define por unas prácticas insuficientes o sedentarias (Tabla 3). A medida que aumenta el nivel de actividad física se incrementan las medias en la Orientación al Ego y Orientación a la Tarea.

\section{Discusión}

Los análisis de varianza realizados para ver si existían diferencias significativas en función del sexo, en las dos dimensiones del TEOSQ-12EF, indicaron que los chicos presentaban una mayor Orientación al Ego en comparación con las chicas, resultado consistente con estudios previos en Educación Física (Flores, Salguero \& Márquez, 2008; Peiró \& Sanchis, 2004; Piéron \& Ruiz-Juan, 2010) y en el deporte (Castillo et al., 2000; Cervelló et al., 1999; Duda, 1988; Duda et al., 1991; Sánchez et al., 2009; White \& Duda, 1994).

Este rechazo de las chicas al ego es coherente con datos de literatura internacional. En un estudio en jóvenes europeos (Ledent, Telama, Cloes, Carvalho \& Piéron, 1996), en todos los países, chicos y chicas desarrollaron una orientación a la tarea más fuerte que la orientación al ego, al igual que sucede en nuestro estudio, algo que se observa también en otros trabajos y perduran en el tiempo (Duda, 1989b).
Esta orientación predominante predice consecuencias favorables sobre participación en actividades físico-deportivas y, sobre todo, para la persistencia y mantenimiento de la práctica físicodeportiva a lo largo de los años. Los individuos orientados a la tarea tienden a atender, para aprender nuevas habilidades, esforzarse y demostrar control del comportamiento. Esta orientación crea un desarrollo personal y refuerza el compromiso para participar en deportes organizados, empleando el tiempo libre de forma activa y saludable.

La orientación al ego, más fuerte en los chicos, puede explicarse por la diferencia entre sexos en elección de actividades. Ellas prefieren actividades de carácter individual, mientras que ellos las eligen colectivas. Esto puede guardar relación con el hecho de que la orientación al ego es una comparación con otros. White y Duda (1994) señalan que los varones dan énfasis al esfuerzo porque es importante para ellos demostrar competencia a través del deporte, alcanzando ciertos niveles de excelencia en una situación donde se evalúa su actuación. Así, a través de esta evaluación por amigos y maestros, los varones pueden mostrar a otros la cantidad de esfuerzo ejercida.

A estas edades, en ambos sexos, se puede concluir que existe una gran estabilidad tanto en la orientación al ego como en la tarea, al corroborarse tanto en nuestro estudio como en otros similares (Peiró \& Sanchis, 2004; Piéron \& Ruiz-Juan, 2010; Ruiz-Juan et al., 2011). Si bien no aparecen diferencias según la edad, entre chicas y chicos del mismo grupo de edad, las comparaciones muestran resultados de ego superiores en ellos. Estos resultados también han sido obtenidos en sujetos norteamericanos y se podría asociar al mayor grado de competitividad observado en chicos (Piéron, 2002).

Según Piéron y Ruiz-Juan (2010), entre los 16 y 20 años, esta estabilidad persiste, aunque se observa una disminución sensible del porcentaje de rechazo. Esta disminución indica una evolución poco deseable cuando se consideran las características y las relaciones de orientación hacia la tarea con otras variables que significan mayor nivel participación, como placer, percepción de competencia y continuación de la actividad en el futuro. Una 
consecuencia de este fenómeno observado podría ser la tendencia al abandono de la práctica.

En nuestro estudio, los activos muestran menor Orientación al Ego $(M=1.81)$ y mayor Orientación a la Tarea $(\mathrm{M}=3.81)$ que los inactivos (abandono y nunca). Igualmente, a medida que aumenta el nivel de actividad física se incrementan las medias en la orientación al ego y la tarea. El abandono podría haber tenido como origen un ego demasiado desarrollado que no ha sido satisfecho por los resultados obtenidos en la práctica y, sobre todo, en las competitivas. Estos fracasos pueden haber conducido a la frustración y, seguidamente, a interrumpir la práctica.

Varios estudios sugieren que la orientación y la percepción del clima implicado en la tarea promueven una actitud, intención positiva y participación hacia el ejercicio (Escarti \& Gutiérrez, 2001; Papaioannou et al., 2004; Wang et al., 2002; Wang et al., 2007). Es conveniente resaltar que el papel del docente de educación física es preponderante, ya que cuando los alumnos perciben que su profesor enfatiza la tarea muestran índices más elevados en dicha orientación y una actitud más positiva, además de pasar más tiempo realizando ejercicio físico (Digelidis et al., 2003; Christodoulidis et al., 2001). Igualmente, que variaciones en los objetivos de logro que pueden tener implicaciones sobre el grado de participación de jóvenes en las actividades físicas (Piéron \& Ruiz-Juan, 2010), algo que es importante para dar indicaciones acerca de pautas para disminuir la prevalencia de inactividad física. Esta evidencia procede de estudios de carácter transversal y longitudinal (Van Mechelen, Twisk, Post, Snel \& Kemper, 1999).

Dempsey, Kimiecik y Horn (1993) y Kimiecik, Horn y Shurin (1996) examinaron en niños la relación de las variaciones de la orientación hacia la tarea con niveles de actividad física de intensidad moderada a fuerte. En estos dos estudios, la orientación hacia la tarea se asociaba de manera positiva con el compromiso en las actividades físicodeportivas. Nuestros resultados son consistentes con estos estudios, incluso muestran congruencia con los resultados de estudios que han utilizado instrumentos objetivos para medir la actividad física dentro de las clases de educación física al revelar que los alumnos con altos valores de orientación a la tarea tuvieron una mayor participación en las actividades físicas vigorosas (Tzetzis et al., 2002).

Varios autores han puesto de relieve que existen relaciones entre orientaciones de meta y continuación de la actividad, intensidad o vigor con el cual realizan ejercicio físico los estudiantes de educación física, deportistas de competición y personas que realizan actividades deportivas en su tiempo libre (Duda, 1988, 1989b; Duda \& Balaguer, 2007; Piéron \& Ruiz-Juan, 2010). En un estudio longitudinal que evaluó la orientación a la tarea y motivación intrínseca tras 7 y 14 meses, se observó que ambas categorías predecían la participación en actividades físicas, mientras que la orientación al ego no se asociaba con la continuidad de la práctica (Papaioannou, Bebetsos, Theodorakis, Christodoulidis \& Kouli, 2006).

Por último, conviene reseñar que el TEOSQ12EF de Ruiz-Juan et al. (2011) es aplicable también en México. Al igual que en la versión original, encontramos que en la estructura factorial aparecen los dos factores (Orientación al Ego y Orientación a la Tarea), que también se hallaron en estudios anteriores en adolescentes deportistas, tanto en la versión inglesa como española (Castillo et al., 2000; Cervelló et al., 1999; Duda, 1989b; Duda, Fox, Biddle \& Armstrong, 1992; Duda \& White, 1992). Como en estudios precedentes, la correlación de Pearson entre ambos factores ha sido bastante buena $(r=0.09)$, con lo cual queda demostrada la independencia de los mismos. Igualmente, la consistencia interna de ambas dimensiones ha sido aceptable $\left(\alpha_{1}=0.76\right.$, Ego; $\alpha_{2}=0.80$, Tarea). El análisis de ítems también aporta adecuados coeficientes de fiabilidad. Estos resultados encuentran apoyo transcultural en este estudio con alumnado mexicano utilizando la adaptación al español del TEOSQ original.

\section{Conclusiones}

Existe una concordancia muy fuerte con los datos de la literatura internacional en este ámbito: predominio de orientación hacia la tarea con relación al 
ego; los alumnos presentan una mayor orientación al ego que las alumnas; son pocas las variaciones que se constatan con la edad, indicándose con ello una estabilidad relativa de las orientaciones. En comparación con el alumnado que nunca ha participado o han abandonado la práctica de actividades físico-deportivas, los que se declaran activos son los que presentan orientaciones principalmente hacia la tarea. Entre los inactivos, la orientación hacia el ego, en porcentajes relativamente elevados, deja prever consecuencias poco favorables de participación en el futuro. De una manera muy destacada, los alumnos cuyo índice de actividad física corresponde a una práctica intensa se orientan hacia la tarea. Estos hallazgos implican que los docentes necesitan crear en sus clases un clima de motivaciones elevado hacia la tarea, para generar una motivación intrínseca que incite a la práctica físico-deportiva en el tiempo libre. Por último, concluir indicando que el TEOSQ-12EF es un instrumento fiable, que reproduce los factores hipotetizados desde el modelo teórico de partida en estudiantes mexicanos.

Para investigaciones futuras, podría ser interesante utilizar instrumentos objetivos para medir el nivel de actividad física dentro de las clases de educación física, realizar programas de intervención utilizando grupos de control y ver la relación con el clima motivacional en las clases, la percepción de competencia y la motivación.

\section{Referencias}

Amado, D., Leo, F., Sánchez, D., González, I. \& López, J. (2012). It is compatible the sport in scholar age with other social roles? A study through Self-Determination Theory. Retos. Nuevas Tendencias en Educación Física, Deporte y Recreación, 21, 50-52.

Ames, C. (1984). Competitive, cooperative and individualistic goal structures: A motivational analysis. In R. Ames \& C. Ames (Eds.), Research on motivation in education: Student motivation (pp.177-207). New York: Academic Press.

Baena, A., Flores, G. \& Barbero, G. (2007). The practice of physical activity and sport in the municipality of environment and their involvement in the curriculum school of physical education. Retos.
Nuevas Tendencias en Educación Física, Deporte y Recreación, 11, 54-59.

Balaguer, I., Castillo, I. \& Tomás, I. (1996). Análisis de las propiedades psicométricas del Cuestionario de Orientación al Ego y a la Tarea en el Deporte (TEOSQ) en su traducción al castellano. Psicológica, 17, 71-81.

Castillo, I., Balaguer, I. \& Duda, J. (2000). Las orientaciones de meta y los motivos de práctica deportiva en jóvenes deportistas valencianos escolarizados. Revista de Psicología del Deporte, 9(1-2), 37-50.

Cervelló, E., Escartí, A. \& Balagué, G. (1999). Relaciones entre la orientación de meta disposicional y la satisfacción con los resultados deportivos, las creencias sobre las causas de éxito en deporte y la diversión con la práctica deportiva. Revista de Psicología del Deporte, 8(1), 7-19.

Cervelló, E., Jiménez, R., Del Villar, F., Ramos, L. \& Santos Rosa, F. (2004). Goal orientations, motivational climate, equality and discipline of Spanish physical education students. Perceptual and Motor Skills, 99(1), 271-283.

Cervelló, E., Moreno, J., Alonso, N. \& Iglesias, D. (2006). Goal orientations, motivational climate and dispositional flow of high school students engaging in extracurricular involvement in physical activity. Perceptual and Motor Skills, 102(1), 87-92.

Chinchilla, J., Escribano, M., Romero, O. \& López, I. (2008). Attitudinal analysis of Physical Education in Compulsory Secondary Education. Retos. Nuevas Tendencias en Educación Física, Deporte y Recreación, 14, 70-74.

Christodoulidis, T., Papaioannou, A. \& Digelidis, N. (2001). Motivational climate and attitudes towards exercise in Greek senior high school: A year-long intervention. European Journal of Sport Science, 1(4), 2-11.

Dempsey, J., Kimiecik, J. \& Horn, T. (1993). Parental influence on children's moderate to vigorous physical activity participation: An expectancy-value approach. Pediatric Exercise Science, 5(2), 151-167.

Digelidis, N., Papaioannou, A., Laparidis, K. \& Christodoulidis, T. (2003). A one-year intervention in 7th grade physical education classes aiming to change motivational climate and attitudes towards exerci- 
se. Psychology of Sport and Exercise, 4(3), 195-210. doi: 10.1016/s1469-0292(02)00002-x

Dishman, R. \& Sallis, J. (1990). Determinants and interventions for physical activity and exercise. En C. Bouchard, R. J. Shephard, T. Stephens, J. R. Sutton \& B. D. McPherson (Eds.), Exercise, fitness and health (pp. 214-235). Champaign, IL: Human Kinetics.

Duda, J. (1988). The relationship between goal perspectives and persistence and intensity among recreational sport participants. Leisure Sciences, 10(2), 95-106.

Duda, J. (1989a). Goal perspectives, participation and persistence in sport. International Journal of Sport Psychology, 20(1), 42-56.

Duda, J. (1989b). The relationship between task and ego orientation and the perceived purpose of sport among male and female high school athletes. Journal of Sport \& Exercise Psychology, 11(3), 318-335.

Duda, J. (1992). Motivation in sport settings: A goal perspective approach. En G. Roberts (Ed.), Motivation in sport and exercise (pp. 57-91). Champaign, IL. Human Kinetics Books.

Duda, J. (1993). Goals: A social-cognitive approach to the study of achievement motivation in sport. En R. N. Murphey \& L. K. Tennant (Eds.), Handbook of research on sport psychology (pp. 421-436). New York: Macmillan.

Duda, J. \& Balaguer, I. (2007). Coach-created motivational climate. En S. Jowet \& D. Lavallee (Eds.), Social Psychology in sport (pp. 117-130). Champaign, IL: Human Kinetics.

Duda, J., Fox, K., Biddle, S. \& Armstrong, N. (1992). Children's achievement goals and beliefs about success in sport. British Journal of Educational Psychology, 62(3), 313-323.

Duda, J. \& Nicholls, J. (1992). Dimensions of achievement motivation in schoolwork and sport. Journal of Educational Psychology, 84(3), 290-299.

Duda, J., Olson, L. \& Templin, T. (1991). The relationship of task and ego orientation to sportsmanship attitudes and the perceived legitimacy of injurious acts. Research Quarterly for Exercise and Sport, 62(1), 79-87.
Duda, J. \& White, S. (1992). Goal orientations and beliefs about the causes of sport success among elite skiers. The Sport Psychologist, 6, 334-343.

Escarti, A. \& Gutiérrez, M. (2001). Influence of the motivational climate in physical education on the intention to practice physical activity or sport. European Journal of Sport Science, 1(4), 1-12.

Ferrer-Caja, E. \& Weiss, M. (2000). Predictors of intrinsic motivation among adolescent students in physical education. Research Quarterly for Exercise and Sport, 71(3), 267-279.

Flores, J., Salguero, A. \& Márquez, S. (2008). Goal orientations and perceptions of the motivational climate in physical education classes among Colombian students. Teaching and Teacher Education, 24(6), 1441-1449.

Gómez, M., Ruiz, F., García, M., Baena, A. \& Granero, A. (2007). Opinion of university students and compulsory Post Secondary Education on the Physical Education classes they have received. Retos. Nuevas Tendencias en Educación Física, Deporte y Recreación, 12, 58-61.

Isorna, M., Rial, A., Vaquero, R. \& Sanmartín, F. (2012). Motivations for the practice of federated sports and canoeing in primary and secondary students. Retos. Nuevas Tendencias en Educación Física, Deporte y Recreación, 21, 19-24.

Kimiecik, J., Horn, T. \& Shurin, C. (1996). Relationships among children's beliefs, perceptions of their parents' beliefs and their moderate-to-vigorous physical activity. Research Quarterly for Exercise and Sport, 67(3), 324-336.

Ledent, M., Telama, R., Cloes, M., Carvalho, L. \& Piéron, M. (1996). Orientation des objectifs et participation à des activités sportives. Sport, $159-$ 160, 82-89.

Lochbaum, M. \& Roberts, G. (1993). Goal orientations and perceptions of the sport experience. Journal of Sport \& Exercise Psychology, 15(2), 160-171.

Molero, D., Ortega, F., Valiente, I. \& Zagalaz, M. (2010). Comparative study based on the physical selfconcept in teenagers regarding gender and physical activity. Retos. Nuevas Tendencias en Educación Física, Deporte y Recreación, 17, 38-41.

Nicholls, J. (1984). Achievement motivation: Conceptions of ability, subjective experience, task 
choice, and performance. Psychological Review, 91(3), 328-346.

Nicholls, J. (1989). Competence and accomplishment: A psychology of achievement motivation. Cambridge, MA: Harvard University Press.

Nicholls, J. (1992). The general and the specific in the development and expression of achievement motivation. En G. C. Roberts (Ed.), Motivation in sport and exercise (pp. 31-57). Champaign, IL: Human Kinetics.

Nicholls, J., Patashnick, M. \& Nolen, S. (1985). Adolescents theories of education. Journal of Educational Psychology, 77(6), 683-692.

Papaioannou, A., Bebetsos, E., Theodorakis, Y., Christodoulidis, T. \& Kouli, O. (2006). Causal relationships of sport and exercise involvement with goal orientations, perceived competence and intrinsic motivation in physical education: A longitudinal study. Journal of Sports Sciences, 24(4), 367-382.

Papaioannou, A., Marsh, H. \& Theodorakis, Y. (2004). A multilevel approach to motivational climate in physical education and sport settings: An individual or a group level construct? Journal of Sport \& Exercise Psychology, 26(1), 90-119.

Peiró, C. \& Sanchis, J. (2004). Las propiedades psicométricas de la versión inicial del cuestionario de orientación a la tarea y al ego (TEOSQ) adaptado a la educación física en su traducción al castellano. Revista de Psicología del Deporte, 13(1), 25-39.

Piéron, M. (2002). Estudi sobre els hàbits esportius dels escolars d'Andorra. Andorra: Govern d'Andorra, Ministeri d'Educació Joventut i Esports.

Piéron, M. \& Ruiz-Juan, F. (2010). Actividad físicodeportiva y salud. Análisis de los determinantes de la práctica en el alumnado de Enseñanza Secundaria. Madrid: Consejo Superior de Deportes.

Raitakari, O., Porkka, K., Taimela, S., Telama, R., Räsänen, L. \& Vikari, J. (1994). Effects of persistent physical activity and inactivity on coronary risk factors in children and young adults. American Journal of Epidemiology, 140(3), 195-205.

Ries, F. (2011). Physical self-concept in adolescents from Seville in relation with gender and the evolution of sport career. Retos. Nuevas Tendencias en Educación Física, Deporte y Recreación, 19, 38-42.
Roberts, G. \& Treasure, D. (1992). Children in sport. Sport Science Review, 1(2), 46-64.

Ruiz-Juan, F., Piéron, M. \& Zamarripa, J. (2011). Versión española del Task and Ego Orientation in Sport Questionnaire (TEOSQ) adaptado a Educación Física. Estudios de Psicología, 32(2), 179-193.

Sánchez, P., Leo, F., Gómez, F., Sánchez, D., de la Cruz, E. \& García, T. (2009). Orientations and motivational climates of the other significatives in young handball players from Extremadura. Retos. Nuevas Tendencias en Educación Física, Deporte y Recreación, 16, 22-27.

Standage, M. \& Treasure, D. (2002). Relationship among achievement goal orientations and multidimensional situational motivation in physical education. British Journal of Educational Psychology, 72(1), 87-103.

Telama, R., Yang, X., Viikari, J., Vãlimãki, I., Wanne, O. \& Raitakari, O. (2005). Physical activity from childhood to adulthood: A 21-year tracking study. American Journal of Preventive Medicine, 28(3), 267-273.

Treasure, D. \& Roberts, G. (1994). Cognitive and affective concomitants of tasks and ego goal orientations during the middle school years. Journal of Sports \& Exercise Psychology, 16(1), 15-28.

Trost, S., Owen, N., Bauman, A., Sallis, F. \& Brown, W. (2002). Correlates of adults' participation in physical activity: Review and update. Medicine and Science in Sports and Exercise, 34(12), 1996-2001.

Tzetzis, G., Goudas, M., Kourteissis, T. \& Zisi, V. (2002). The relation of goal orientations to physical activity in physical education. European Physical Education Review, 8(2), 177-188.

Van Der Horst, K., Paw, M., Twisk, J. \& Van Mechelen, W. (2007). A brief review on correlates of physical activity and sedentariness in youth. Medicine and Science in Sports and Exercise, 39(8), 1241-1250.

Van Mechelen, W., Twisk, J., Post, B., Snel, J. \& Kemper, H. (1999). Physical activity of young people: The Amsterdam longitudinal growth and health study. Medicine and Science in Sports and Exercise, 32(9), 1610-1616.

Walling, M., Duda, J. \& Chi, L. (1993). The perceived motivational climate in sport questionnaire: 
Construct and predictive validity. Journal of Sport \& Exercise Psychology, 15(2), 172-183.

Wang, J., Biddle, S. \& Elliot, A. (2007). The $2 \times 2$ achievement goal framework in a physical education context. Psychology of Sport and Exercise, 8(2), 147168. doi: 10.1016/j.psychsport.2005.08.012

Wang, J., Chatzisarantis, N., Spray, C. \& Biddle, S. (2002). Achievement goal profiles in school physical education: Differences in self-determination, sport ability beliefs, and physical activity. British Journal of Educational Psychology, 72(3), 433-445. White, S. \& Duda, J. (1994). The relationship of gender, level of sport involvement, and participation motivation to task and ego orientation. International Journal of Sport Psychology, 25(1), 4-18.

Williams, L. \& Gill, D. (1995). The Role of Perceived Competence in the Motivation of Physical Activity. Journal of Sport \& Exercise Psychology, 17(4), 363-378. 
